\title{
"Envelope" approach for onlay bone grafting: Preliminary surgical and prosthetic results
}

\author{
Federico Hernández-Alfaro ${ }^{1}$, Eva Salvan-Garcia ${ }^{2}$, Javier Mareque-Bueno ${ }^{3}$, Eduard Ferres-Padró ${ }^{4}$
}

\author{
${ }^{1} \mathrm{MD}$, DDS, PhD, FEBOMS. Clinical Professor Oral and Maxillofacial Surgery, Director Master in Implant Dentistry, Univer- \\ sitat Internacional de Catalunya. Chief Department OMFS, Teknon Medical Center, Barcelona, Spain \\ ${ }^{2}$ DDS. Associate Professor in Oral Implantology. Universitat Internacional de Catalunya, Barcelona, Spain \\ ${ }^{3} \mathrm{MD}$, DDS, PhD. Associate Professor Oral and Maxillofacial Surgery, Universitat Internacional de Catalunya. Barcelona, \\ Spain \\ ${ }^{4}$ MD, DDS, PhD. Clinical Professor Oral and Maxillofacial Surgery, Director Department Oral Surgery. Universitat Interna- \\ cional de Catalunya, Barcelona, Spain
}

Correspondence:

C/ Vilana, 12, D-185.

Barcelona, 08022, Spain.

director@institutomaxilofacial.com

Received: 05/12/2009 Accepted: 07/04/2010

\begin{abstract}
Hernández-Alfaro F, Salvan-García E, Mareque-Bueno J, Ferres-Padró E. "Envelope" approach for onlay bone grafting: Preliminary surgical and prosthetic results. Med Oral Patol Oral Cir Bucal. 2011 Jan 1;16 (1):e45-9.

http://www.medicinaoral.com/medoralfree01/v16i1/medoralv16ilp45.pdf
\end{abstract}

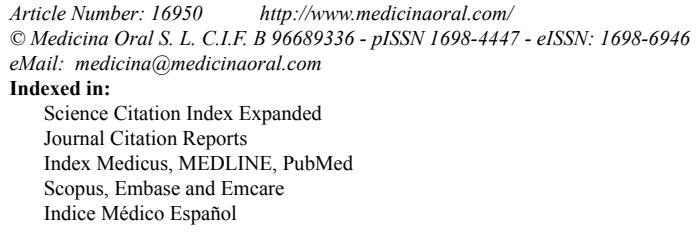

Indice Médico Español

\begin{abstract}
Minimally invasive surgery principles have been applied to preprosthetic-implant surgery, and an adequate presurgical planning reduces morbidity in both harvesting and stabilization of the bone grafts. Objective: A new "envelope" approach for onlay bone grafting at the alveolar process is presented, this avoids releasing incisions yet allowing adequate reconstruction of the defective site. Study design: To be included in the study the patient had to have an antero-posterior defect of the alveolar crest. Patient age and sex, location of the defect, type and size of graft, and complications appeared during follow up were recorded. Results: Ten consecutive patients (4 men and 6 women) have been succesfully treated with this technique between June/2006 and February/2008. Eight defects were located at the anterior maxilla (between canines), and the remaining two at the anterior mandible (between canines). Four patients received bone grafts harvested from the chin, and the remaining six patients received bone grafts from the mandibular body. Mean size of the graft was: width 11,5mm (range 8 to 17), height 14,7mm (range 9 to 18), depth 4,3mm (range 3 to 6). Conclusion: This technique improves vascularization of the mucoperiosteal flap and avoidance of releasing incisions which has an aesthetic impact in patients with gingival smile.
\end{abstract}

Key words: Preprosthetic surgery, bone grafts, implants, aesthetic zone, flap design. 


\section{Introduction}

Implant therapy in the anterior maxilla is challenging for the clinician because of the aesthetic demands of patients and difficult pre-existing anatomy. A through analysis is mandatory to evaluate the extent of the defect and determine whether the missing tissue is hard or soft in nature. Rosenquist defined 4 factors considered important to achieve a successful and aesthetically satisfying results; these are: width and position of the attached gingiva, buccal contour of the alveolar process, level and configuration of the alveolar process, and size and shape of the papillae (1). Adequate soft tissue closure represents a critical factor in the success of preprosthetic surgery as well as implant placement, especially when simultaneous peri-implant bone augmentation is performed (1-6). In cases of bone atrophy, a number of techniques have been proposed depending on the location and size of the defect (1,7-9), and different methods have been proposed for management of soft tissues $(2,9,10)$.

Bone grafting techniques for alveolar preprosthetic reconstruction have been well documented in the literature with variable degrees of success $(1,7,8,11,12)$. A number of different materials such as autogenous grafts, allografts or alloplastic grafting materials have been used, however the vast author majority report the superiority of autogenous bone for alveolar reconstruction $(12,13)$, and the absolute necessity for preserving adequate soft tissue coverage when bone augmentation is performed $(1,4)$. Autogenous bone is considered the gold standard for osseous reconstruction because it does not produce immunologic reactions and it contains osteoinductive components $(9,11-16)$. Both extraoral $(1,3,13)$ and intraoral $(2,5,7-13,17)$ donor sites have been proposed. Lately minimally invasive surgery principles have been applied to preprosthetic-implant surgery, and an adequate presurgical planning might reduce morbidity in both harvesting and stabilization of the bone grafts (1).

Basic biological principles favour the use of well vascularized flaps to cover reconstructed areas. The rationale is to maximize the blood supply to the underlying graft, and to prevent ischemic changes at the distal portions of the flap which could eventually lead to suture dehiscence $(6,9,18)$.

A new "envelope" approach for onlay bone grafting at the alveolar process is presented, which avoids releasing incisions yet allowing adequate reconstruction of the defective site.

\section{Material and Methods}

- Patient Selection

Ten consecutive patients with inadequate bone volume for implant placement have been prospectively treated with this technique between June/2006 and February/2008 by the same maxillofacial surgeon (FHA) in the Department of Oral and Maxillofacial Surgery at General Hospital of Catalonia and Teknon Medical Center, Barcelona, Spain. To be included in the study the patient had to have an antero-posterior defect of the alveolar crest and couldn't be a smoker. All patients were informed and had to sign an appropriate consent form.

Patient age and sex, location of the defect, type and size of graft and complications appeared during follow up were recorded.

Prior treatment in all patients a 3D CT scan was made and used to define the volume and pattern of the defect and thus simulate the shape and size of the graft needed. - Surgical technique

In this study prophylactic oral antibiotics were used (Amoxicillin 500mg) beginning 8 hours prior to the procedure and continuing for 7 days. Both donor and recipient sites were infiltrated with local anaesthetic ( $2 \%$ lidocaine with adrenaline 1:100.000). With a $\mathrm{n}^{\circ} .15$ blade, a through-and-through incision was made at the alveolar crest of the defective site. The papillae of the neighbouring teeth were included in the incision, and this continued in the sulcus of these teeth up to the most apical point of the gingival margin. No releasing incisions were made.

Blind and sharp subperiosteal dissection of the flap was then performed with a small periosteal elevator, and a subperiosteal pocket was developed (Fig 1. a,b). To

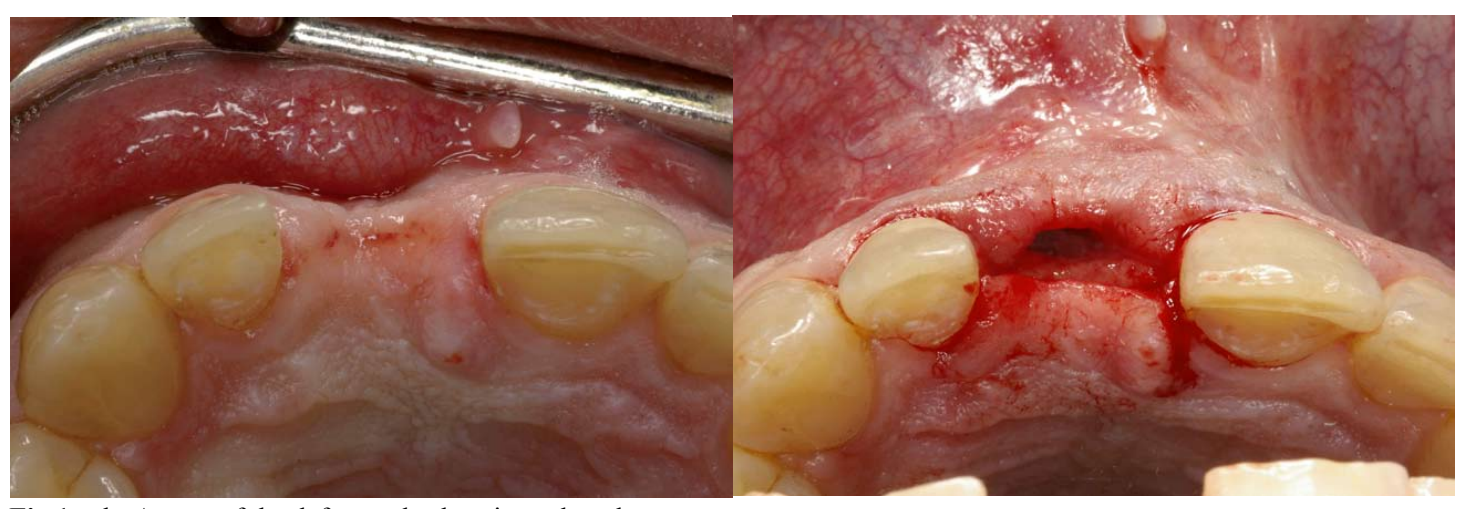

Fig 1. a,b Aspect of the defect and subperiosteal pocket. 
prevent tension in the flap after placement of the graft, horizontal releasing incisions were made at the periosteum in a blind fashion.

Although pre-measured in the CT scan, the defect was evaluated with a perio probe. At this point the donor site, chin or mandibular body was approached. The graft was harvested with the aid of a piezoelectric saw and a chisel, and after remodelling of the graft it was fixed at the recipient site with a single 1,5 screw placed in a compression fashion (Fig 2). In three cases the screw entered at the most coronal part of the graft, and in the remaining seven, it was placed transmucosally.

In four cases, particulated material was added and a resorbable membrane (bio-gide, Geislicht) was used to cover the reconstruction (Fig 3a). Finally the flap was repositioned and interrupted 5/0 vicryl or nylon sutures were placed (Fig 3b). Postsurgery, 500mg amoxicillin every 8 hours for 7 days and $0.02 \%$ clorhexidine mouthwash were prescribed. Three months later, the screw was removed and a transmucosal dental implant was placed. After verifying the stability of the implant, the appropriate abutment was selected and the provisional crown was made. The occlusion was checked because provisional crown should avoid centric and excursive contacts.

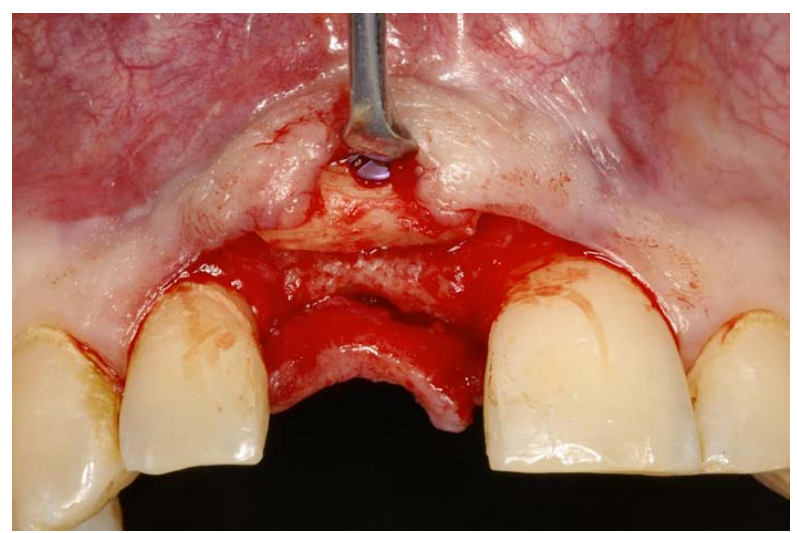

Fig 2. Placement and fixation of the graft with a single screw via coronal approach and placed in a compression fashion.

\section{Results}

Six women and four men were treated during the period analyzed. Mean age was 33 (range 18 to 62). Eight defects were located at the anterior maxilla (between canines), and the remaining two at the anterior mandible (between canines).

From an overall of 10 patients 4 received bone grafts harvested from the chin, whereas 6 of them received bone graft from the mandibular body. Grafts mean size was: width $11,5 \mathrm{~mm}$ (range 8 to 17 ), height $14,7 \mathrm{~mm}$ (range 9 to 18), depth 4,3mm (range 3 to 6).

All the screws used in this study to fix the grafts were 1,5 in diameter and 7 to $10 \mathrm{~mm}$ in length.

One case presented partial dehiscence at the sutures and healed spontaneously over the underlying membrane.

\section{Discussion}

Pre-implant reconstruction of the alveolar crest is mandatory when insufficient bone volume and/or quality is present, because the underlying bone structure plays a key role in the establishment of esthetic soft tissues in the anterior maxilla. A number of site development procedures have been proposed to achieve optimal alveolar architecture $(1,7,8,13,17)$. Bone grafting has proved to be a predictable technique when performed with sound consideration to bone and soft tissue biology $(1,9)$. In our study block-type grafts were harvested from the chin and the body area due to their benefits: minimal resorption and early revascularization attributed to its embryologic origin $(9,14)$ and better incorporation at the donor site $(9,11)$. We used a barrier membrane in four cases to help contain and stabilize the particulate graft, to allow bony regeneration at any void space, and to minimize the overall volume loss. But variable degrees of resorption must be considered, so overgrafting is indicated. Resorption of the onlay grafts varies from one patient to another, and it occurs mainly on the buccal aspect of the graft.

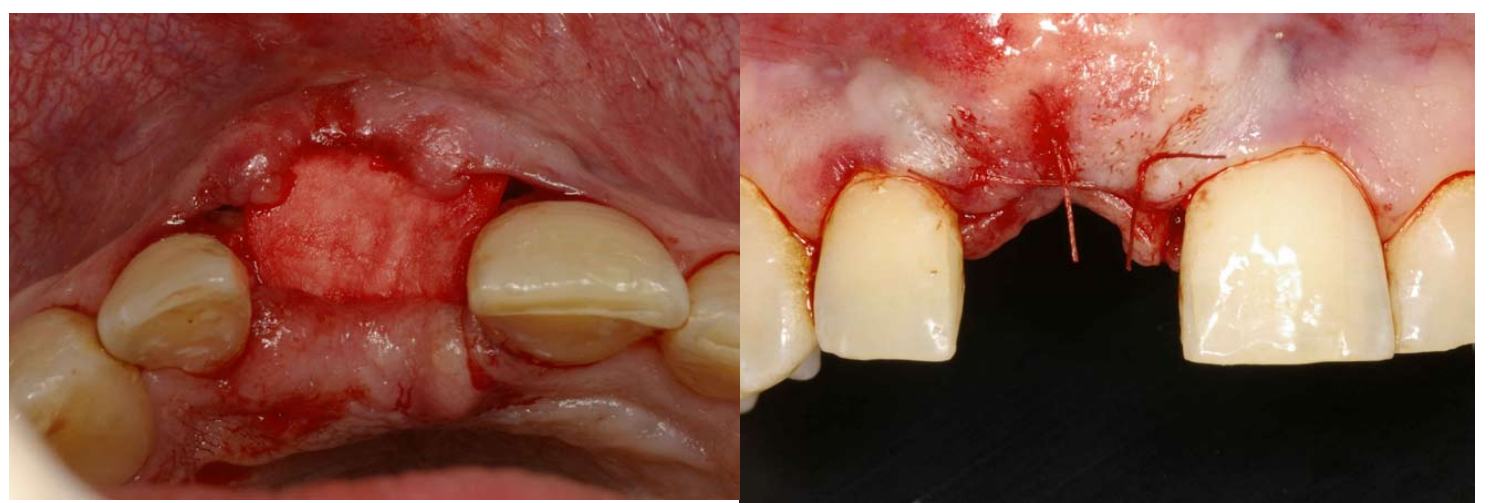

Fig 3. a,b Covering of the reconstruct with a resorbable membrane and tension-free repositioning of the flap with interrupted $5 / 0$ sutures. 
Preservation and incorporation of the graft depends largely on adaptation, fixation and coverage by well vascularized flaps. The bone graft and the recipient site should be sculpted to obtain close approximation of the surfaces $(1,4)$. Fixation of the bone graft to the recipient site is accomplished using self-tapping titanium miniscrews, the recommended diameter of the fixation screw is between 1.3 and $1.6 \mathrm{~mm}$. Initial stabilization of the graft is crucial, because absolute immobilization is necessary for the complete healing of the bone graft without a fibrous component (7-9). In all likelihood, the etiology of the incision-line breakdown is the result of a blood supply compromised by preexisting poorly vascularized tissue or by closure of the flap under tension $(1,4,6)$. Incising the periosteum at the base of the flap allows a tension-free adaptation of the wound margins $(5,6,8,9,17)$.

Classically, vertical or oblique releasing incisions have been made in the flaps to gain access to a defective site. The rationale was to have a clear view of the defect and to ease adaptation and fixation of the graft, and to allow advancement of the flap for closing $(6,7,18)$.

Modern preprosthetic planning includes accurate CT diagnosis of the defects and planning of the amount and pattern of the needed grafts before actually performing surgeries $(1,8-10,17,19)$. This means that it is not always necessary to visualize the defect provided that a through radiological analysis has been made beforehand.

According to Kleinheinz(6) the main course of the supplying arteries is from posterior to anterior and main vessels run parallel to the alveolar ridge in the vestibulum. For these reasons releasing incisions in the vestibulum should be avoided because they will cut obliquely through defined esthetic zones and not at their borders (6).

Moreover, in cases were previous surgeries have been made with high horizontal incisions; nutrition to the flaps comes from the buccal corridors. If releasing incisions interrupts these, vascularization of the flap might be impaired and there are chances for ischemic necrosis of the flap and dehiscence over the bone graft.

Another characteristic of vascularization is that the crestal area of the edentulous alveolar ridge is covered by an avascular zone with no anastomoses crossing the alveolar ridge, because of it, the midcrestal incision in the edentulous area of the alveolar ridge seems the safest and most reliable incision in this situation $(6,18)$.

The marginal incision including the papilla is the incision recommended especially for esthetically problematic areas in the upper incisor region (6). The most frequently reported and feared complications with bone grafting in the literature are incision dehiscence, exposure of the block grafts and bone resorption $(1,4,20)$. Overextending the graft thickness can prevent bone resorption. Exposure of the block graft during healing could take place even if a membrane is not used. The time of the exposure may be the key factor that determines the fate of the graft. Revascularization is an ongoing process, and it is unknown when an intramembranous bone graft will completely heal. Early exposure occurring at a time when the graft material has not yet been revascularized can result in susceptibility to infection. Late bone graft exposure may not necessarily result in graft necrosis or prevent graft integration (9). In all these circumstances it seems logical that maintaining widely pedicled flaps by eliminating the lateral releasing incisions would aid in preventing complications. In the present study the only complication was partial dehiscence at the sutures in one case which healed spontaneously over the underlying membrane.

This "envelope" approach for alveolar bone grafting is specially important at the anterior maxilla in patients with gingival smile, because releasing incisions often leave visible scars which compromise the aesthetic results.

At suturing time the absence of releasing incisions did not show a decrease in wound closure, and not advancing the flap preserves the height of the keratinized mucosa in the area and the position of the neighbouring papillae.

As a conclusion, despite the short follow-up and small number of patients, this preliminary report shows the feasibility and advantages of a new "envelope approach". This technique improves vascularization of the mucoperiosteal flap and avoidance of releasing incisions which has an aesthetic impact in patients with gingival smile.

\section{References}

References with links to Crossref - DOI

1. Bahat O, Fontanessi RV. Efficacy of implant placement after bone grafting for three-dimensional reconstruction of the posterior jaw. Int J Periodontics Restorative Dent. 2001;21:220-31.

2. Triaca A, Minoretti R, Merli M, Merz B. Periosteoplasty for soft tissue closure and augmentation in preprosthetic surgery: a surgical report. Int J Oral Maxillofac Implants. 2001;16:851-6.

3. Hernández-Alfaro F, Martí C, Biosca MJ, Gimeno J. Minimally invasive tibial bone harvesting under intravenous sedation. J Oral Maxillofac Surg. 2005;63:464-70.

4. Bahat O, Fontanesi FV. Complications of grafting in the atrophic edentulous or partially edentulous jaw. Int J Periodontics Restorative Dent. 2001;21:487-95.

5. Tecimer D, Behr MM. Use of autogenous bone grafting to reconstruct a mandibular knife edge ridge before implant surgery: a case report. J Oral Implantol. 2001;27:98-102.

6. Kleinheinz J, Büchter A, Kruse-Lösler B, Weingart D, Joos U. Incision design in implant dentistry based on vascularization of the mucosa. Clin Oral Implants Res. 2005;16:518-23.

7. Bedrossian E, Tawfilis A, Alijanian A. Veneer grafting: a technique for augmentation of the resorbed alveolus prior to implant placement. A clinical report. Int J Oral Maxillofac Implants. 2000;15:853-8.

8. Sethi A, Kaus T. Ridge augmentation using mandibular block bone grafts: preliminary results of an ongoing prospective study. Int J Oral Maxillofac Implants. 2001;16:378-88.

9. Capelli M. Autogenous bone graft from the mandibular ramus: a technique for bone augmentation. Int $\mathrm{J}$ Periodontics Restorative Dent. 2003;23:277-85. 
10. Hernández-Alfaro F, Pages CM, García E, Corchero G, Arranz C. Palatal core graft for alveolar reconstruction: a new donor site. Int $\mathrm{J}$ Oral Maxillofac Implants. 2005;20:777-83.

11. Proussaefs P, Lozada J, Kleinman A, Rohrer MD. The use of ramus autogenous block grafts for vertical alveolar ridge augmentation and implant placement: a pilot study. Int J Oral Maxillofac Implants. 2002;17:238-48.

12. McCarthy C, Patel RR, Wragg PF, Brook IM. Dental implants and onlay bone grafts in the anterior maxilla: analysis of clinical outcome. Int J Oral Maxillofac Implants. 2003;18:238-41.

13. Kainulainen VT, Sàndor GK, Carmichael RP, Oikarinen KS. Safety of zygomatic bone harvesting: a prospective study of 32 consecutive patients with simultaneous zygomatic bone grafting and 1-stage implant placement. Int $J$ Oral Maxillofac Implants. 2005;20:245-52.

14. Proussaefs P, Lozada J. The use of intraorally harvested autogenous block grafts for vertical alveolar ridge augmentation: a human study. Int J Periodontics Restorative Dent. 2005;25:351-63.

15. Gapski R, Wang HL, Misch CE. Management of incision design in symphysis graft procedures: a review of the literature. J Oral Implantol. 2001;27:134-42.

16. Schuler R, Verardi S. A new incision design for mandibular symphysis bone-grafting procedures. J Periodontol. 2005;76:845-9.

17. Schwartz-Arad D, Levin L. Intraoral autogenous block onlay bone grafting for extensive reconstruction of atrophic maxillary alveolar ridges. J Periodontol. 2005;76:636-41.

18. Koymen R, Karacayli U, Gocmen-Mas N, Ertugrul-Koymen C, Ortakoglu K, Gunaydin Y, et al. Flap and incision design in implant surgery: clinical and anatomical study. Surg Radiol Anat. 2009;31:301-6.

19. Wheeler SL. Implant complications in the esthetic zone. J Oral Maxillofac Surg. 2007;65(7 Suppl 1):93-102. Review. Erratum in: J Oral Maxillofac Surg. 2008;66:2195-6.

20. Li J, Wang HL. Common implant-related advanced bone grafting complications: classification, etiology, and management. Implant Dent. 2008;17:389-401. 